\title{
A Survey Paper on Context Base Routing Protocol (CBRP) in IoT
}

\author{
Dr. Pardeep Kumar \\ Associate Professor. \\ Department of Computer Science and Engineering. \\ G H Raisoni College of Engineering, Nagpur, India
}

\begin{abstract}
The unique Internet structure was no longer designed to meet contemporary demands and the continuous pressure in terms of state-of-the-art threats, performance, reliability, scalability and security. The quantities of data which might be created these days require ever-developing amounts of storage. However, records isn't inherently associated with expertise in their context. This information may additionally be available at the conversation end-points (applications) but now not when facts is in transit. So, it is not viable to make efficient storage choices that assure fast storage control, fast information mining and retrieval, clean and removal optimized for different facts.

The lack of context-aware control is closely associated with the lack of consumer and facts privacy. In CBRP (Context Based Routing Protocol) for Internet of Things, primarily based on context-focus, which belongs to the key technology of Internet of Things.
\end{abstract}

Keywords: IoT, Context, Protocol

\section{INTRODUCTION}

According to Moore's law [1], the storage restrict of computational devices and their processing electricity double approximately every years and their length decreases. This phenomenon started to fast development of small clever gadgets that have sources and strength to collect, process, and share data on their own. Nowadays human beings can get smart cars, fridges, TVs, which have a more computational capability and can resolve more complex and important obstacles through themselves. It moreover requires it is moreover for them to talk with every other to share information. This tendency emerged in so-called "The Internet of Things" (IoT) paradigm, which means different devices with various features to be connected to a community and offer feasibility for better clarifications for every day human dilemmas. Figure 1.1 depicts a general gadget of the IoT

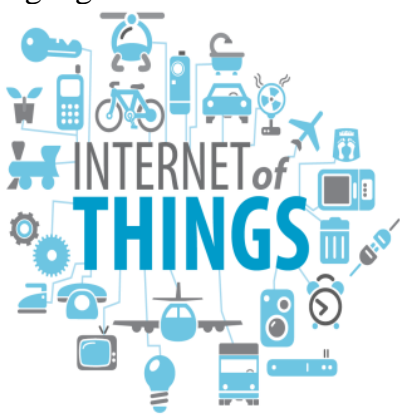

Figure 1.1: General system of Internet of Things [2]

The author was introduced[3] IoT definition as "Interconnection of sensing and actuating mechanisms giving the ability to share data across platforms through a unified framework, developing a standard operating picture for enabling innovative applications".

It achieved with the aid of seamless large-scale sensing, information analytics and data illustration using present day ubiquitous sensing and cloud computing .

This definition gives a deep understanding of the paradigm but also implies possible significant hurdles in designing IoT applications and systems. Because of the quantity of operating data, such structures require sizeable garage, processing, and verbal exchange resources. In [3] it's miles declared that records garage is an important difficulty inside the era of IoT. Therefore, more intelligent methods and strategies for coping with this amount of facts are highly required.

For the past few years studies network addressed these challenges. One of the created strategies, to overcome them, is context-aware computing [4]. It is a technique to machine development, which entails utilisation of any meaningful statistics (referred to as context), retrieved from the gadgets. It might comprise the statistics about the user's location, innovative time, user's activity, and customers surrounding environment. So information may enhance the IoT-primarily based structures. Context recognition gives an opportunity for integration and repurposing the IoT statistics across a couple of systems, platforms, and programs by using context facts linked to sensor information [5]. It can also make it easier to perform communique between gadgets in IoT primarily based structures [5]. The [6] confirmed that context-conscious computing might reduce energy consumption and bandwidth in different non-stop and uploading sensing programs. In IoT network when massive numbers of sensor and motive device are positioned and its generation massive range of the statistics. The traditional deviceoriented utility approach turns into unworkable, seeing that all the complicated responsibility regarding the control of IoT gadgets and their statistics becomes an utility role which can deliver scalability issues for massive-scale scenarios. The many researchers [7] introduced to mitigate this inefficiency, widespread quantities of middleware solutions.

Another feature of middleware systems is the contextawareness that is a challenge of this area according to [7]. The moving towards fame of IoT the number of sensor nodes is expanding slowly It is a commonplace experience that these sensors will generate a tremendous quantity of data [8], and, unless analyze, interpret, and apprehend these data, they'll maintain useless and with no meaning to 
enhance the society.Context-aware is an essential role because the significant number of the data is available then it process takes more time. It is challenging in the previous network such as mobile internet [7].

In context-aware situations, there is a cycle for the conception of the information that will address to the users, which comprises data acquisition, information modelling, reasoning, and sharing or distribution of contextualized information. Thus, the architecture of a context-aware machine should be capable of offer offerings for the combination and automation of all levels of the facts cycle. These paintings aimed to broaden a system mechanism to provide context-aware facts offerings to IoT environments. It attaches this mechanism to an IoT middleware system, which is the reference platform used in this work [9].

\section{LITERATURE REVIEW}

The routing protocol in IoT and WSN are taken care of in two types for the communique of the nodes including complanate routing protocols and hierarchical. The complanate routing protocols recognize at the shortest path to the vacation spot node however hierarchical need the assist of the overall routing protocol working inside the cluster and it's also looked after on the bottom of the records and region information.

\subsection{Routing Protocols in IoT}

In IoT, it connects every device to the Internet, and it comprises some application in different environments to improve the quality of the lives, and this application will create a significant amount of data. The one shortcoming of the developing subject is the creation of an unusual amount of data, its storage, ownership, security, expiry and its miles routing to the preferred destination. The routing in IoT it's far the large project as it consists of the different community and it's far more and more tough for low strength and lossy radio-links, multi-hop mesh topologies, battery supplied nodes are regularly changed due to the community topologies [10].The device in the network will intercommunicate among each other, and some device is moving and active elements because of that many issues arise in the evolution of routing protocol, and it becomes challenging. According to the author [11], the full functionality of IoT and sensible protocols are required for D2D communique in IoT. It requires the efficient and scalable routing protocols adaptable to unique eventualities and network size variations and capable of finding.

\subsection{Context-Awareness in Routing}

The author [12] described the 5 W's inclusive of Who, What, Where, When and Why this is used for the getting the context. It refers the statistics accumulated from the distinctive source to as because the raw information, and before amassing the context, it's far necessary to realize the functions of context for efficaciously describing it. It having a few values associated with them for describing specific attributes and best identification and preprocessing of the context records is not sufficient also need to hold the satisfactory of the context. QoC is evaluated by a few parameters, including the accuracy of the context and validity of the context. The environment of the context adjustments time to time due to all the device is mobile so it is vital to have greater accurate, legitimate and unexpired context constantly to take the proper decision. It divides the context dissemination into two exceptional thought such as first; the context is stored in context server, and it saves other in a distributed way both these methods are having wonderful and poor aspects. According to the researchers, $2 \mathrm{~d}$ is greater significant because in a first case every time the node needs to speak with the context server for doing a particular activity, and it requires extra energy. Some demanding situations are related to the context-aware routing described in below. Context acquisition and distribution: For smart routing, it is necessary to collect the context from the environment. In the IoT, network topology has to gather the raw information from various sensors and to pre-process and to validate the information getting the exact context information.

* Context quality: The author [13] define the parameters of the best of the context comprising context records validity, context accuracy, and up-to-datedness' of context information, and it also said that first-rate of the context depends upon the physical sensors, facts and transport process.

- Context storage: It is also challenging undertaking to keep the context due to the shortage of memory. It can save the context in two methods such as save the context inside the compressed form, and another technique is to shop the context within the centrally situated, i.e. Context server.

Some blessings of context-conscious routing is such as intelligent routing, community load balancing, community lifetime maximization, and discount in verbal exchange delay.

\section{1] Energy aware Ant Routing Algorithm (EARA)}

This EARA ambitions to evolve routing manner for maximizing the community lifetime [14], and it is a swarm intelligence or bio-stimulated set of rules. In the Ant Routing Algorithm (ARA), ant agents of EARA hold the records of additional fields such as a

a) The average electricity of the nodes by a few hops a packet travelled.

b) It also stores a lowest residual electricity value minimal which an ant agent encounters within the path.

It uses the agents for updating the electricity values within the nodes routing table, and it broadcasted PEANTs on the vacation spot accumulate the energy statistics on that path, and it is very luxurious operation in order that the algorithm sends these management packets occasionally. In EARA, the destination node keeping song of the remaining electricity of their battery and the adjustments in energy in keeping with the configurable threshold then EARA broadcast the message inside the community with new PEANT, or it's far referred to as marketers. In the PEANT (periodic strength ant retailers) time $c$ language of broadcasting depends on two parameters, i.e. most battery capacity and the threshold cost of energy which is configured. 


\section{Virtue in EARA:}

It is context-conscious routing protocol and multi-hop.

\section{Shortcomings in EARA:}

i. Security of statistics is not considered.

ii. The threshold value of the alternate in power may affect the performance of it.

\section{2] Routing protocol over low power and lossy networks $(\mathrm{RPL})$ :}

It develops this protocol via International Engineering Task Force (IETF) for low electricity and lossy networks, and it's far taken into consideration as a de facto routing standard for the Internet of Things having the aim to optimize the routing scheme for converge cast visitors pattern. Routing is very tough for 6LoWPAN networks due to the low strength and lossy radio links, the battery furnished nodes, multi-hop mesh topologies and common topology changes because of mobility and its miles' distance vector protocol. It constructs a DestinationOriented Directed Acyclic Graph (DODAG) the usage of one or several metrics, and the DODAG is generated by way of considering the hyperlink costs, node attributes and an aim function. The rank era for every node at the DODAG is performed by the goal function, and it helps various types of visitors together with multi-point to factor, factors to multi-point and factor to point. It is a loopunfastened topology, and the rank should strictly monotonically growth from the root towards the leaves of the DODAG.

They split it into many partitions depending upon the utility of the context so in this situation it may form over one uncoordinated DODAG's with unbiased roots and multiple times of RPL can run simultaneously on the network devices. In this RPLInstanceID is used for the unique identification of the instance. DODAG Information Option (DIO) messages which are used for the formation and maintenance of the network topologies, and it broadcast the message multi-cast periodically and connect to every node in the network for establishing a path towards the root node. The message comprises the facts inclusive of the DODAG identifier, the aim function, the rank of the node, or the metrics used for the direction calculation. After receiving the DIO message, the neighboring node can set its rank based on its neighbor's rank. The Destination Advertisement Object (DAO) messages are used to lower back, propagate the routing information from leaf nodes to the roots.

\section{Virtue in RPL:}

i. It does now not require translation gateways for gaining access to the nodes within the network from out-of-doors world it's miles based on the stop to cease IP.

Ii. It dynamically adapts the sending fee of the routing manipulate messages if you want to regularly be generated only if the network is in unstable condition.

Iii. It permits optimization of the community for one of a kind application scenarios and deployment.

\section{Shortcomings in RPL:}

i. It does no longer aid multipath routing.

ii. In IoT network the electricity balancing and cargo

balancing aren't taken into consideration.

\section{3] Multiparent routing in RPL:}

The motive of designing this routing protocol is to maximize the overall lifetime of the community through taking care of most electricity confined nodes, i.E. Bottlenecks. It takes a lifetime of the community into consideration because the period earlier than the dying of the primary node of the community because of run out of the electricity. The author [15] Oana Iova et al. Proposed the Expected Lifetime (ELT) metric for denoting the more time of the node. It is constructed a DODAG based on ELT metric for accurately estimating the life of all the routes toward the border router and designed a mechanism for detecting bottlenecks for spreading the site visitors load to several parents. In this network node uses all its parents, assigning a weight of traffic to every of them and distributes the strength consumption equitably among all the paths toward the border router. Only part of its traffic will ultimately arrive at a selected bottleneck, and the strength intake is well balanced.

\section{Virtue in multiparent routing in RPL:}

i. It supports the multipath routing to enhance the fault tolerance, congestion avoidance and satisfactory of the service.

ii. It also will increase the community lifetime by way of balancing the traffic load amongst multiple parents.

\section{4] PAIR (Pruned Adaptive IoT Routing):}

The creator [4] define the IoT network, and it belongs to one of a kind owner, PAIR it's far pricing version which helps the intermediate nodes to get some monetary blessings as they utilize their assets for relaying. The pricing of the node depends upon a few parameters together with residual power and electricity consumption Current load and buffer space. In PAIR distance to neighbor works in stages which includes ahead and backwards. The creator centered on the ahead's stage, in these message are broadcasted through the source to its buddies which contain the price visible from the source to the modern node and the intermediate nodes gain those messages, they forward them to their acquaintances through updating the price based on a fixed of parameters. The vacation spot node sends the acknowledgement (ack) at the best-selected direction based totally at the gathered values of value parameters from the setup message. During the acknowledgement message a breaks in the path at an innovative node $i$ and then it's far converted into the setup message, or it is known as as stop and it ahead to the friends for finding the route. The supply node gets the setup message the active direction receives hooked up among the supply and vacation spot, and the records transmission might also get started. At the time of the statistics transmission if the hyperlink is broken then either it completes the transmission of the facts at the alternate route or by buffering the received facts, stop messages are generated for coming across a new course in the destination's direction.

\section{Virtue in PAIR:}

i. It is multihop and contexts-aware routing protocol. 
ii. It solves the handshaking between the nodes of the heterogeneous community via looking to give a few incentives to the depending node.

\section{Shortcomings in PAIR:}

i. it does not consider The security attributes.

ii. It requires extra memory compared to buffer capability if the alternate route is finding whilst they accrue the hyperlink ruin within the communication.

\section{5] REL (Routing protocol based on Energy and Link Quality):}

During the path choice procedure it uses the link first-rate of wireless hyperlinks besides the residual power for growing the device reliability and first-class of the provider to the gadget. The wi-fi hyperlink is unreliable when they are facing problem inclusive of low-strength radios and sensitivity to noise, interference and multipath distortions. The analysing the link is relied upon the link satisfactory Indicator. REL stores and workable routes towards the destination and select the one based on

i) excellent of wi-fi links primarily based on weak hyperlinks metric

ii) ii)residual energy and

iii) iii) hop remember to avoid lengthy and inefficient paths.

The selection of a path depends upon the two thresholds: hop remember threshold HCdiff_max - permit and power threshold Eth. In this focused on the power threshold and it's far used inside the route selection and load balancing mechanism. The Eth corresponds to the tracking of energy degrees in each node individually. It calculates the distinction between modern $\mathrm{E}(\mathrm{t})$ and formerly recorded $\mathrm{E}(\mathrm{t}$ - 1) energy level. The difference is extra than the brink then-advisor message gives the statistics about the new price of residual electricity to neighbouring nodes, and if the low-price show it consumes the energy.

\section{Virtue in REL:}

i. For the hyperlink to the routing, it considers the link of satisfactory.

ii. The hyperlink quality is good the extra risk of successful packet delivery, which saves greater electricity.

iii. They avoid the immoderate use of a single route or unmarried node because of the load balancing mechanism.

\section{6] Context Base Routing Protocol (CBRP)}

It divides CBRP node in the network into cluster area based on the field dimension, numbers of node in the field and the number of rounds. In the cluster area decide the one cluster head based on the energy and threshold value or node which is nearer to the base station and every time the cluster head is change. The Context-Aware in our work decides according to the user activity, location, time.
The following table 2.1 shows the parameter base comparison of protocol in IoT.

\begin{tabular}{|l|l|l|l|l|l|l|}
\hline & $\begin{array}{l}\text { Conte } \\
\text { xt } \\
\text { Pwar } \\
\text { entocol }\end{array}$ & $\begin{array}{l}\text { Secur } \\
\text { e }\end{array}$ & $\begin{array}{l}\text { Multi- } \\
\text { hop } \\
\text { routin } \\
\text { g }\end{array}$ & $\begin{array}{l}\text { Suppor } \\
\text { ts } \\
\text { dynami } \\
\text { c } \\
\text { topolog } \\
\text { y }\end{array}$ & $\begin{array}{l}\text { Incentiv } \\
\text { e } \\
\text { based }\end{array}$ & $\begin{array}{l}\text { Conside } \\
\text { rs } \\
\text { Link } \\
\text { quality }\end{array}$ \\
\hline EARA & Yes & No & Yes & & No & No \\
\hline RPL & Yes & Yes & Yes & Yes & No & No \\
\hline $\begin{array}{l}\text { Multi- } \\
\text { parent } \\
\text { routing } \\
\text { in RPL }\end{array}$ & Yes & No & Yes & Yes & No & No \\
\hline PAIR & Yes & No & Yes & Yes & Yes & No \\
\hline REL & Yes & No & Yes & & No & Yes \\
\hline CBRP & Yes & Yes & Yes & Yes & Yes & Yes \\
\hline
\end{tabular}

\section{CONCLUSION AND FUTURE SCOPE}

In an IoT network, nodes have more potent sensory potential and smarter intelligence. So in this paper CBRP as the representative which using the core concept of the context-conscious computing into the basis routing protocol. Context statistics becomes extra important, because we can not satisfy by using collecting the data, however understanding the meaning of the data. The Context is relied on the consumer because some researchers are determined the context of consumer, activity, time and location.

In destiny we can prove the context base routing protocol is advanced to the alternative protocol in IoT with the assist of the some practical parameters.

\section{REFERENCES}

[1] Robert R Schaller. Moore's law: past, present and future. IEEE spectrum, 34(6):52-59,1997.

[2] Ilex Content Strategies Ltd. Addressing the security in the Internet of Things. (2017, May 12) [Blog]. Available: http://www.ilexcontent.com/addressing-security-in-the-internetofthings- iot/

[3] Jayavardhana Gubbi, Rajkumar Buyya, Slaven Marusic, and Marimuthu Palaniswami. Internet of things (iot): A vision, architectural elements, and future directions. Future generation computer systems, 29(7):1645-1660, 2013.

[4] Gregory D Abowd, Anind K Dey, Peter J Brown, Nigel Davies, Mark Smith, and Pete Steggles. Towards a better understanding of context and context-awareness. In International Symposium on Handheld and Ubiquitous Computing, pages 304-307. Springer, 1999.

[5] Charith Perera, Arkady Zaslavsky, Peter Christen, and Dimitrios Georgakopoulos. Context aware computing for the internet of things: A survey. IEEE Communications Surveys \& Tutorials, 16(1):414-454, 2014.

[6] Wanita Sherchan, Prem P Jayaraman, Shonali Krishnaswamy, Arkady Zaslavsky, Seng Loke, and Abhijat Sinha. Using on-themove mining for mobile crowdsensing. In Mobile Data Management (MDM), 2012 IEEE 13th International Conference on, pages 115124. IEEE, 2012.

[7] Pardeep Kumar, Udayabhanu N P G Raju, "Protected Combination Of Artificial Neural Network With Wireless Sensor Networks", International Journal of Engineering and Advanced Technology (IJEAT), vol. 8, Issue. 4, pp. 08 - 13, April 2019. ISSN:2249 8958

[8] F. Neto and C. Ribeiro, "Dynamic change of services in wireless sensor network middleware based on semantic technologies," in Autonomic and Autonomous Systems (ICAS), 2010 Sixth International Conference on, march 2010, pp. 58-63. [Online]. Available: http://dx.doi.org/10.1109/ICAS.2010.17

[9] G. D. Abowd, A. K. Dey, P. J. Brown, N. Davies, M. Smith, and P. Steggles, "Towards a better understanding of context and contextawareness," in Proceedings of the 1st international symposium on 
Handheld and Ubiquitous Computing, ser. HUC '99. London, UK: Springer-Verlag, 1999, pp. 304-307. [Online]. Available: http://dl.acm.org/citation.cfm?id=647985.743843

[10] Sharief M. A. Oteafy, Fadi M. Al-Turjman and Hossam S. Hassanein, "Pruned Adaptive Routing in the Heterogeneous Internet of Things", Global Communications Conference (GLOBECOM), 2012 IEEE, pp. 214 -219, ISSN 1930-529X.

[11] Oladayo Bello, Sherali Zeadally, "Intelligent Device-to-Device Communication in the Internet of Things

[12] G. D. Abowd and E. D. Mynatt, "Charting past, present, and future research in ubiquitous computing," ACM Trans. Comput.-Hum. Interact., vol. 7, pp. 29-58, March 2000.

[13] P. Bellavista, A. Corradi, M. Fanelli, and L. Foschini, "A survey of context data distribution for mobile ubiquitous systems," ACM Computing Surveys, vol. xx, no. xx, p. 49, 2013.

[14] Michael Frey, Friedrich Grose, Mesut Gunes, "Energy-aware Ant Routing in Wireless Multi-hop Networks", IEEE International Conference on Communications (ICC), pp. 190-196, 2014.

[15] Oana Iova, Fabrice Theoleyre, Thomas Noel, "Using Multiparent Routing in RPL to Increase The Stability and The Lifetime of The Network", Ad Hoc Networks, Elsevier 2015, pp. 45-62. 